\title{
Additive Non-Approximability of Chromatic Number in Proper Minor-Closed Classes
}

\author{
Zdeněk Dvořák ${ }^{1}$ \\ Charles University, Malostranske namesti 25, 11800 Prague, Czech Republic \\ rakdver@iuuk.mff.cuni.cz \\ (iD) https://orcid.org/0000-0002-8308-9746
}

\section{Ken-ichi Kawarabayashi ${ }^{2}$}

National Institute of Informatics, 2-1-2 Hitotsubashi, Chiyoda-ku, Tokyo 101-8430, Japan

k_keniti@nii.ac.jp

(D) https://orcid.org/0000-0001-6056-4287

\begin{abstract}
Robin Thomas asked whether for every proper minor-closed class $\mathcal{G}$, there exists a polynomialtime algorithm approximating the chromatic number of graphs from $\mathcal{G}$ up to a constant additive error independent on the class $\mathcal{G}$. We show this is not the case: unless $\mathrm{P}=\mathrm{NP}$, for every integer $k \geq 1$, there is no polynomial-time algorithm to color a $K_{4 k+1}$-minor-free graph $G$ using at most $\chi(G)+k-1$ colors. More generally, for every $k \geq 1$ and $1 \leq \beta \leq 4 / 3$, there is no polynomial-time algorithm to color a $K_{4 k+1}$-minor-free graph $G$ using less than $\beta \chi(G)+(4-3 \beta) k$ colors. As far as we know, this is the first non-trivial non-approximability result regarding the chromatic number in proper minor-closed classes.

We also give somewhat weaker non-approximability bound for $K_{4 k+1}$-minor-free graphs with no cliques of size 4 . On the positive side, we present an additive approximation algorithm whose error depends on the apex number of the forbidden minor, and an algorithm with additive error 6 under the additional assumption that the graph has no 4-cycles.
\end{abstract}

2012 ACM Subject Classification Mathematics of computing $\rightarrow$ Graph theory

Keywords and phrases non-approximability, chromatic number, minor-closed classes

Digital Object Identifier 10.4230/LIPIcs.ICALP.2018.47

\section{Introduction}

The problem of determining the chromatic number of a graph, or even of just deciding whether a graph is colorable using a fixed number $c \geq 3$ of colors, is NP-complete [7], and thus it cannot be solved in polynomial time unless $\mathrm{P}=\mathrm{NP}$. Even the approximation version of the problem is hard: for every $\varepsilon>0$, Zuckerman [16] proved that unless $\mathrm{P}=\mathrm{NP}$, there exists no polynomial-time algorithm approximating the chromatic number of an $n$-vertex graph within multiplicative factor $n^{1-\varepsilon}$.

There are more restricted settings in which the graph coloring problem becomes more tractable. For example, the well-known Four Color Theorem implies that deciding $c$ colorability of a planar graph is trivial for any $c \geq 4$; still, 3-colorability of planar graphs is NP-complete [7]. From the approximation perspective, this implies that chromatic number of planar graphs can be approximated in polynomial time up to multiplicative factor of $4 / 3$ (but not better), and additively up to 1 .

1 Supported by project 17-04611S (Ramsey-like aspects of graph coloring) of Czech Science Foundation. 2 Supported by JST ERATO Grant Number JPMJER1305, Japan. 
Let $\Sigma$ be a fixed surface, and consider a graph $G$ embedded in $\Sigma$. If $G$ is large enough, Euler's formula implies that its average degree is less than 7 , and thus $G$ contains a vertex $v$ of degree at most 6 . Removing $v$ and repeating this observation, we conclude that the vertex set of $G$ can be partitioned in parts $A$ and $B$ such that $A$ has bounded size (depending only on $\Sigma)$ and $G[B]$ is 6 -degenerate. We can now by brute force find an optimal coloring of $G[A]$ and color $G[B]$ greedily using at most 7 additional colors, thus obtaining a proper coloring of $G$ using at most $\chi(G[A])+7 \leq \chi(G)+7$ colors. That is, for any fixed surface $\Sigma$, there exists a linear-time algorithm to approximate chromatic number of graphs embedded in $\Sigma$ with additive error at most 7 , independent of the surface (this additive error can be improved to 2 using the fact that $c$-colorability of embedded graphs can be decided in polynomial-time for $c \geq 5$ based on a deep result of Thomassen [14]).

If a graph can be drawn in a given surface, all its minors can be drawn there as well. Hence, it is natural to also consider the coloring problem in the more general setting of proper minor-closed classes. Motivated by the simple algorithm for embedded graphs from the previous paragraph (and the minor structure theorem [12], which relates proper minorclosed classes to embedded graphs), Robin Thomas [13] conjectured that such an additive approximation algorithm exists for graphs from any proper minor-closed class.

- Conjecture 1. For some fixed constant $\alpha \geq 0$, the following holds: for every proper minor-closed class $\mathcal{G}$, there exists a polynomial-time algorithm taking as an input a graph $G \in \mathcal{G}$ and returning an integer $c$ such that $\chi(G) \leq c \leq \chi(G)+\alpha$.

The fact that we do not allow $\alpha$ depend on the class $\mathcal{G}$ is important: any $K_{k}$-minor-free graph is $O(k \sqrt{\log k})$-colorable [10], and thus its chromatic number can be trivially approximated with additive error $O(k \sqrt{\log k})$.

Since it is easy to decide whether $\chi(G)<3$, Conjecture 1 would imply that chromatic number in proper minor-closed classes can be approximated up to a fixed multiplicative factor (at most $1+\alpha / 3$ ). And indeed, this weaker statement holds: As shown by DeVos et al. [4] and algorithmically by Demaine et al. [2], for every proper minor-closed class $\mathcal{G}$, there exists a constant $\gamma_{\mathcal{G}}$ such that the vertex set of any graph $G \in \mathcal{G}$ can be partitioned in polynomial time into two parts $A$ and $B$ with both $G[A]$ and $G[B]$ having tree-width at most $\gamma_{\mathcal{G}}$. Consequently, $\chi(G[A])$ and $\chi(G[B])$ can be determined exactly in linear time [1], and we can color $G[A]$ and $G[B]$ using disjoint sets of colors, obtaining a coloring of $G$ using at most $\chi(G[A])+\chi(G[B]) \leq 2 \chi(G)$ colors.

In the light of this evidence, Conjecture 1 seems quite plausible. Kawarabayashi et al. [9] conjectured that it actually holds even in a stronger form, in the list coloring setting. As our main result, we show that Conjecture 1 is actually false, even if we allow further multiplicative error by a factor smaller than $4 / 3$. A graph $F$ is $t$-apex if there exists a set $X$ of at most $t$ vertices of $F$ such that $F-X$ is planar.

- Theorem 2. Let $k_{0}$ be a positive integer, let $F$ be a $\left(4 k_{0}-3\right)$-connected graph that is not $\left(4 k_{0}-4\right)$-apex, and let $1 \leq \beta \leq 4 / 3$ be a real number. Unless $P=N P$, there is no polynomial-time algorithm taking as an input an $F$-minor-free graph $G$ and returning an integer $c$ such that $\chi(G) \leq c<\beta \chi(G)+(4-3 \beta) k_{0}$.

In particular, in the special case of $\beta=1$ and $F$ being a clique, we obtain the following.

- Corollary 3. Let $k_{0}$ be a positive integer. Unless $P=N P$, there is no polynomial-time algorithm taking as an input a $K_{4 k_{0}+1}$-minor-free graph $G$ and returning an integer $c$ such that $\chi(G) \leq c \leq \chi(G)+k_{0}-1$. 
On the positive side, Kawarabayashi et al. [9] showed it is possible to approximate the chromatic number of $K_{k}$-minor free graphs in polynomial time additively up to $k-2$. We leave open the question whether a better additive approximation (of course above the bound $\approx k / 4$ given by Corollary 3 ) is possible. Another positive result was given by Demaine et al. [3], who proved that if $H$ is a 1-apex graph, then the chromatic number of $H$-minor-free graphs can be approximated additively up to 2 . Let us also remark that if $H$ is 0 -apex (i.e., planar), then $H$-minor-free graphs have bounded tree-width [11], and thus their chromatic number can be determined exactly in linear time [1].

The apex number of a graph $F$ is the minimum $t$ such that $F$ is $t$-apex. Theorem 2 shows that the additive error of any approximation algorithm for chromatic number of $F$-minor-free graphs must depend on the apex number of $F$. On the positive side, we show that such algorithm exists, generalizing the results from the previous paragraph.

- Theorem 4. Let $t$ be a positive integer and let $H$ be a t-apex graph. There exists a polynomial-time algorithm taking as an input an $H$-minor-free graph $G$ and returning an integer $c$ such that $\chi(G) \leq c \leq \chi(G)+t+3$.

The construction we use to establish Theorem 2 results in graphs with large clique number (on the order of $k_{0}$ ). On the other hand, forbidding triangles makes the coloring problem for embedded graphs more tractable - all triangle-free planar graphs are 3-colorable [8] and there exists a linear-time algorithm to decide 3-colorability of a triangle-free graph embedded in any fixed surface [6]. It is natural to ask whether Conjecture 1 could not hold for triangle-free graphs, and this question is still open. On the negative side, we show that forbidding cliques of size 4 is not sufficient.

- Theorem 5. Let $\beta$ and $d$ be real numbers such that $1 \leq \beta<4 / 3$ and $d \geq 0$. Let $m=\lceil d /(4-3 \beta)\rceil$. There exists a positive integer $k_{0}=O\left(m^{4} \log ^{2} m\right)$ such that the following holds. Let $F$ be a $\left(4 k_{0}-1\right)$-connected graph with at least $4 k_{0}+8$ vertices that is not $\left(4 k_{0}-4\right)$ apex. Unless $P=N P$, there is no polynomial-time algorithm taking as an input an $F$-minorfree graph $G$ with $\omega(G) \leq 3$ and returning an integer $c$ such that $\chi(G) \leq c<\beta \chi(G)+d$.

In particular, in the special case of $\beta=1$ and $F$ being a complete graph, we get the following.

- Corollary 6. For every positive integer $k_{0}$, there exists an integer $d=\Omega\left(k_{0}^{1 / 4} / \log ^{1 / 2} k_{0}\right)$ as follows. Unless $P=N P$, there is no polynomial-time algorithm taking as an input a $K_{4 k_{0}+8^{-}}$ minor-free graph $G$ with $\omega(G) \leq 3$ and returning an integer $c$ such that $\chi(G) \leq c \leq \chi(G)+d$.

On the positive side, we offer the following small improvement to the additive error of Theorem 4 .

Theorem 7. Let $t$ be a positive integer and let $H$ be a $t$-apex graph. There exists a polynomial-time algorithm taking as an input an $H$-minor-free graph $G$ with no triangles and returning an integer $c$ such that $\chi(G) \leq c \leq \chi(G)+\lceil(13 t+172) / 14\rceil$.

What about graphs of larger girth? It turns out that Conjecture 1 holds for graphs of girth at least 5 , with $\alpha=6$. Somewhat surprisingly, it is not even necessary to forbid triangles to obtain this result, just forbidden 4-cycles are sufficient. Indeed, we can show the following stronger result.

Theorem 8. Let $a \leq b$ be positive integers and let $\mathcal{G}$ be a proper minor-closed class of graphs. There exists a polynomial-time algorithm taking as an input a graph $G \in \mathcal{G}$ not containing $K_{a, b}$ as a subgraph and returning an integer $c$ such that $\chi(G) \leq c \leq \chi(G)+a+4$. 
Let us remark that the multiplicative 2-approximation algorithm of Demaine et al. [2] can be combined with the algorithms of Theorems 4, 7 , and 8 by returning the minimum of their results. E.g., if $H$ is a $t$-apex graph, then there is a polynomial-time algorithm coloring an $H$-minor-free graph $G$ using at most $\min (2 \chi(G), \chi(G)+t+3) \leq \beta \chi(G)+(2-\beta)(t+3)$ colors, for any $\beta$ such that $1 \leq \beta \leq 2$; the combined multiplicative-additive non-approximability bounds of Theorems 2 and 5 are also of interest in this context.

The rest of the paper is organized as follows. In Section 2, we present a graph construction which we exploit to obtain the non-approximability results in Section 3. The approximation algorithms are presented in Section 4.

\section{Tree-like product of graphs}

Let $G$ and $H$ be graphs, and let $|V(G)|=n$ and $V(H)=\left\{u_{1}, \ldots, u_{k}\right\}$. Let $T_{n, k}$ denote the rooted tree of depth $k+1$ such that each vertex at depth at most $k$ has precisely $n$ children (the depth of the tree is the number of vertices of a longest path starting with its root, and the depth of a vertex $x$ is the number of vertices of the path from the root to $x$; i.e., the root has depth 1). For each non-leaf vertex $x \in V\left(T_{n, k}\right)$, let $G_{x}$ be a distinct copy of the graph $G$ and let $\theta_{x}$ be a bijection from $V\left(G_{x}\right)$ to the children of $x$ in $T_{n, k}$. If $v \in V\left(G_{x}\right), y$ is a non-leaf vertex of the subtree of $T_{n, k}$ rooted in $\theta_{x}(v)$, and $z \in V\left(G_{y}\right)$, then we say that $v$ is a progenitor of $z$. The level of $v$ is defined to be the depth of $x$ in $T_{n, k}$. Note that a vertex at level $j$ has exactly one progenitor at level $i$ for all positive $i<j$. The graph $T(G, H)$ is obtained from the disjoint union of the graphs $G_{x}$ for non-leaf vertices $x \in V\left(T_{n, k}\right)$ by, for each edge $u_{i} u_{j} \in E(H)$ with $i<j$, adding all edges from vertices of $T(G, H)$ at level $j$ to their progenitors at level $i$. Note that the graph $T(G, H)$ depends on the ordering of the vertices of $H$, which we consider to be fixed arbitrarily.

Let $Q_{1}$ and $Q_{2}$ be graphs, containing cliques $K_{1}$ and $K_{2}$ of the same size $k$. A graph $Q$ is a clique-sum of graphs $Q_{1}$ and $Q_{2}$ on cliques $K_{1}$ and $K_{2}$ if $Q$ is obtained from $Q_{1}$ and $Q_{2}$ by identifying the two cliques and possibly removing some edges of the resulting clique. We use the following well-known observation: If $F$ is a $(k+1)$-connected graph and $F$ is a minor of $Q$, then $F$ is also a minor of $Q_{1}$ or $Q_{2}$.

- Lemma 9. Let $G$ and $H$ be graphs with $|V(G)|=n \geq 2$ and $V(H)=\left\{u_{1}, \ldots, u_{k}\right\}$. Let $q \leq k$ be the maximum integer such that $\left\{u_{k-q+1}, \ldots, u_{k}\right\}$ is an independent set in $H$. The graph $T(G, H)$ has $O\left(n^{k}\right)$ vertices and $\omega(T(G, H))=\omega(G)+\omega(H)-1$. Furthermore, if $F$ is a minor of $T(G, H)$ and $F$ is $(k-q+1)$-connected, then there exists a set $X \subseteq V(F)$ of size at most $k-q$ such that $F-X$ is a minor of $G$.

Proof. The tree $T_{n, k}$ has $1+n+n^{2}+\ldots+n^{k-1} \leq 2 n^{k-1}$ non-leaf vertices, and thus $|V(T(G, H))| \leq 2 n^{k}$.

Consider a clique $K$ in $T(G, H)$, and let $v$ be a vertex of $K$ of largest level. Let $x$ be the vertex of $T_{n, k}$ such that $v \in V\left(G_{x}\right)$. Note that all vertices of $K \backslash V\left(G_{x}\right)$ are progenitors of $v$, and the vertices of $H$ corresponding to their levels are pairwise adjacent. Consequently, $\left|K \backslash V\left(G_{x}\right)\right| \leq \omega(H)-1$ and $\left|K \cap V\left(G_{x}\right)\right| \leq \omega(G)$. Therefore, each clique in $T(G, H)$ has size at most $\omega(G)+\omega(H)-1$. A converse argument shows that cliques $K_{G}$ in $G$ and $K_{H}$ in $H$ give rise to a clique in $T(G, H)$ of size $\left|K_{G}\right|+\left|K_{H}\right|-1$, implying that $\omega(T(G, H))=\omega(G)+\omega(H)-1$.

For $i=0, \ldots, k-q$, let $G_{i}$ denote the graph obtained from $G$ by adding $i$ universal vertices. Observe that $T(G, H)$ is obtained from copies of $G_{0}, \ldots, G_{k-q}$ by clique-sums on cliques of size at most $k-q$ (corresponding to the progenitors whose level is most $k-q$ ). Hence, each $(k-q+1)$-connected minor $F$ of $T(G, H)$ is a minor of one of $G_{0}, \ldots, G_{k-q}$, and thus a minor of $G$ can be obtained from $F$ by removing at most $k-q$ vertices. 
For an integer $p \geq 1$, the $p$-blowup of a graph $H_{0}$ is the graph $H$ obtained from $H_{0}$ by replacing every vertex $u$ by an independent set $S_{u}$ of $p$ vertices, and by adding all edges $z z^{\prime}$ such that $z \in S_{u}$ and $z^{\prime} \in S_{u^{\prime}}$ for some $u u^{\prime} \in E\left(H_{0}\right)$. For the purposes of constructing the graph $T(G, H)$, we order the vertices of $H$ so that for each $u \in V\left(H_{0}\right)$, the vertices of $S_{u}$ are consecutive in the ordering. Note that $\chi(H) \leq \chi\left(H_{0}\right)$, since we can assign all the vertices of $S_{u}$ the color of $u$.

The strong $p$-blowup of $H_{0}$ is obtained from the $p$-blowup by making the sets $S_{u}$ into cliques for each $u \in V\left(H_{0}\right)$. For integers $a \geq b \geq 1$, an $(a: b)$-coloring of $H_{0}$ is a function $\varphi$ that to each vertex of $H_{0}$ assigns a subset of $\{1, \ldots, a\}$ of size $b$ such that $\varphi(u) \cap \varphi(v)=\emptyset$ for each edge $u v$ of $H_{0}$. The fractional chromatic number $\chi_{f}\left(H_{0}\right)$ is the infimum of $\left\{a / b: H_{0}\right.$ has an $(a: b)$-coloring $\}$. Note that if $H$ is the strong $p$-blowup of a graph $H_{0}$, then a $c$-coloring of $H$ gives a $(c: p)$-coloring of $H_{0}$. Consequently, we have the following.

- Observation 10. If $H$ is the strong $p$-blowup of a graph $H_{0}$, then $\chi(H) \geq p \chi_{f}\left(H_{0}\right)$.

We now state a key result concerning the chromatic number of the graph $T(G, H)$.

- Lemma 11. Let $p, c \geq 1$ be integers and let $G$ be a graph. Let $H_{0}$ be a graph such that $\chi\left(H_{0}\right)=\chi_{f}\left(H_{0}\right)=c$, and let $H$ be the p-blowup of $H_{0}$. Then

$$
\chi(T(G, H)) \leq c \chi(G)
$$

and if $\chi(G) \geq p$, then

$$
\chi(T(G, H)) \geq c p .
$$

Proof. Let $V(H)=\left\{u_{1}, \ldots, u_{k}\right\}$, where $k=p\left|V\left(H_{0}\right)\right|$. Note that $\chi(H) \leq \chi\left(H_{0}\right)=c$. Let $\varphi_{H}$ be a proper coloring of $H$ using $c$ colors. Let $C_{1}, \ldots, C_{c}$ be pairwise disjoint sets of $\chi(G)$ colors. For each non-leaf vertex $x$ of $T_{n, k}$ of depth $i$, color $G_{x}$ properly using the colors in $C_{\varphi_{H}\left(u_{i}\right)}$. Observe that this gives a proper coloring of $T(G, H)$ using at most $c \chi(G)$ colors, and thus $\chi(T(G, H)) \leq c \chi(G)$.

Suppose now that $\chi(G) \geq p$ and consider a proper coloring $\varphi$ of $T(G, H)$. We construct a path $P=x_{1} x_{2} \ldots x_{k+1}$ in the tree $T_{n, k}$ from its root $x_{1}$ to one of the leaves and a coloring $\psi$ of $H$, as follows. Suppose that we already selected $x_{1}, \ldots, x_{i}$ for some $i \leq k$. Let $Z_{i}$ denote the set of progenitors of level at least $i-p+1$ of the vertices of $G_{x_{i}}$. Since $\left|Z_{i}\right| \leq p-1$ and $\varphi$ uses at least $\chi(G) \geq p$ distinct colors on $G_{x_{i}}$, there exists $v \in V\left(G_{x_{i}}\right)$ such that $\varphi(v)$ is different from the colors of all vertices of $Z_{i}$. We define $x_{i+1}=\theta_{x_{i}}(v)$ be the child of $x_{i}$ in $T_{n, k}$ corresponding to $v$, and set $\psi\left(u_{i}\right)=\varphi(v)$.

Note that $\psi$ is a proper coloring of $H$ such that for each $u \in V\left(H_{0}\right), \psi$ assigns vertices in $S_{u}$ pairwise distinct colors. Consequently, $\psi$ is a proper coloring of the strong $p$-blowup of $H_{0}$, and thus $\psi$ (and $\varphi$ ) uses at least $p \chi_{f}\left(H_{0}\right)=c p$ distinct colors by Observation 10. We conclude that $\chi(T(G, H)) \geq c p$.

For positive integers $p$ and $k$, let $K_{k \times p}$ denote the $p$-blowup of $K_{k}$, i.e., the complete $k$-partite graph with parts of size $p$. Let us summarize the results of this section in the special case of the graph $T\left(G_{0}, K_{k \times 4}\right)$ with $G_{0}$ planar.

- Corollary 12. Let $G_{0}$ be a planar graph with $n$ vertices and let $k_{0}$ be a positive integer. Let $G=T\left(G_{0}, K_{k_{0} \times 4}\right)$. The graph $G$ has $O\left(n^{4 k_{0}}\right)$ vertices. If $G_{0}$ is 3 -colorable, then $\chi(G) \leq 3 k_{0}$, and otherwise $\chi(G) \geq 4 k_{0}$. Furthermore, every $\left(4 k_{0}-3\right)$-connected graph appearing as a minor in $G$ is $\left(4 k_{0}-4\right)$-apex. 
Proof. Note that $\chi\left(K_{k_{0}}\right)=\chi_{f}\left(K_{k_{0}}\right)=k_{0},\left|V\left(K_{k_{0} \times 4}\right)\right|=4 k_{0}$ and the last 4 vertices of $K_{k_{0} \times 4}$ form an independent set. The claims follow from Lemma 9 (with $H=K_{k_{0} \times 4}, k=4 k_{0}$ and $q=4$, using the fact that every minor of a planar graph is planar) and Lemma 11 (with $H_{0}=K_{k_{0}}, p=4$ and $\left.c=k_{0}\right)$.

\section{Non-approximability}

The main non-approximability result is a simple consequence of Corollary 12 and NP-hardness of testing 3-colorability of planar graphs.

Proof of Theorem 2. Suppose for a contradiction that there exists such a polynomial-time algorithm $\mathcal{A}$, taking as an input an $F$-minor-free graph $G$ and returning an integer $c$ such that $\chi(G) \leq c<\beta \chi(G)+(4-3 \beta) k_{0}$.

Let $G_{0}$ be a planar graph, and let $G=T\left(G_{0}, K_{k_{0} \times 4}\right)$. By Corollary 12 , the size of $G$ is polynomial in the size of $G_{0}$ and $G$ is $F$-minor-free. Furthermore, if $G_{0}$ is 3-colorable, then $\chi(G) \leq 3 k_{0}$, and otherwise $\chi(G) \geq 4 k_{0}$. Hence, if $G_{0}$ is 3 -colorable, then the value returned by the algorithm $\mathcal{A}$ applied to $G$ is less than $\beta \chi(G)+(4-3 \beta) k_{0} \leq 4 k_{0}$, and if $G_{0}$ is not 3 -colorable, then the value returned is at least $\chi(G) \geq 4 k_{0}$. This gives a polynomial-time algorithm to decide whether $G_{0}$ is 3-colorable.

However, it is NP-hard to decide whether a planar graph is 3-colorable [7], which gives a contradiction unless $\mathrm{P}=\mathrm{NP}$.

Note that the graphs $T\left(G_{0}, K_{k_{0} \times 4}\right)$ used in the proof of Theorem 2 have large cliques (of size greater than $k_{0}$ ). This turns out not to be essential - we can prove somewhat weaker non-approximability result even for graphs with clique number 3 . To do so, we need to apply the construction with both $G$ and $H_{0}$ being triangle-free. A minor issue is that testing 3-colorability of triangle-free planar graphs is trivial by Grötzsch' theorem [8]. However, this can be worked around easily.

- Lemma 13. Let $\mathcal{G}$ denote the class of graphs such that all their 3-connected minors with at least 12 vertices are planar. The problem of deciding whether a triangle-free graph $G \in \mathcal{G}$ is 3-colorable is NP-hard.

Proof. Let $R_{0}$ be the Grötzsch graph ( $R_{0}$ is a triangle-free graph with 11 vertices and chromatic number 4 , and all its proper subgraphs are 3 -colorable). Let $R$ be a graph obtained from $R_{0}$ by removing any edge $u v$. Note that $R$ is 3 -colorable and the vertices $u$ and $v$ have the same color in every 3 -coloring.

Let $G_{1}$ be a planar graph. Let $G_{2}$ be obtained from $G_{1}$ by replacing each edge $x y$ of $G_{1}$ by a copy of $R$ whose vertex $u$ is identified with $x$ and an edge is added between $v$ and $y$ (i.e., $G_{2}$ is obtained from $G_{1}$ by a sequence of Hajós sums with copies of $\left.R_{0}\right)$. Clearly, $G_{2}$ is trianglefree, it is 3-colorable if and only if $G_{1}$ is 3-colorable, and $\left|V\left(G_{2}\right)\right|=\left|V\left(G_{1}\right)\right|+10\left|E\left(G_{1}\right)\right|$. Furthermore, $G_{2}$ is obtained from a planar graph by clique-sums with $R_{0}$ on cliques of size two, and thus every 3-connected minor of $G_{2}$ is either planar or a minor of $R_{0}$ (and thus has at most 11 vertices). Hence, $G_{2}$ belongs to $\mathcal{G}$.

Since testing 3-colorability of planar graphs is NP-hard, it follows that testing 3-colorability of triangle-free graphs from $\mathcal{G}$ is NP-hard.

We also need a graph $H_{0}$ which is triangle-free and its fractional chromatic number is large and equal to its ordinary chromatic number. Such a graph can be obtained by a standard probabilistic argument (omitted). 
- Lemma 14. For every positive integer $m$, there exists a triangle-free graph $H_{m}$ with $O\left(m^{4} \log ^{2} m\right)$ vertices such that $\chi\left(H_{m}\right)=\chi_{f}\left(H_{m}\right)=m$.

Theorem 5 now follows in the same way as Theorem 2, using the graphs from Lemma 14 instead of cliques.

Proof of Theorem 5. Suppose for a contradiction that there exists such a polynomial-time algorithm $\mathcal{A}$, taking as an input an $F$-minor-free graph $G$ with $\omega(G) \leq 3$ and returning an integer $c$ such that $\chi(G) \leq c<\beta \chi(G)+d$. Recall that $m=\lceil d /(4-3 \beta)\rceil$. Let $H_{m}$ be the graph from Lemma 14. Let $k_{0}=\left|V\left(H_{m}\right)\right|$ and let $H$ be the 4-blowup of $H_{m}$. Let $\mathcal{G}$ be the class of graphs such that all their 3 -connected minors with at least 12 vertices are planar.

Consider a triangle-free graph $G_{0} \in \mathcal{G}$, and let $G=T\left(G_{0}, H\right)$. By Lemma 9 , the size of $G$ is polynomial in the size of $G_{0}$. Consider any $\left(4 k_{0}-1\right)$-connected minor $F^{\prime}$ of $G$. By Lemma 9 , there exists a set $X$ of size at most $4 k_{0}-4$ such that $F^{\prime}-X$ is a minor of $G_{0}$. Since $F^{\prime}-X$ is 3-connected and $G_{0} \in \mathcal{G}$, we conclude that either $\left|V\left(F^{\prime}\right)\right| \leq|X|+11 \leq 4 k_{0}+7$ or $F^{\prime}-X$ is planar. Consequently, $F^{\prime} \neq F$, and thus $G$ does not contain $F$ as a minor. Furthermore, $\omega(G) \leq \omega\left(G_{0}\right)+\omega(H)-1 \leq 3$.

Recall that $\chi\left(H_{m}\right)=\chi_{f}\left(H_{m}\right)=m$. By Lemma 11, if $G_{0}$ is 3-colorable, then $\chi(G) \leq 3 m$, and otherwise $\chi(G) \geq 4 \mathrm{~m}$. Hence, if $G_{0}$ is 3 -colorable, then the value returned by the algorithm $\mathcal{A}$ applied to $G$ is less than $\beta \chi(G)+d \leq 3 \beta m+d \leq 4 m$, and if $G_{0}$ is not 3-colorable, then the value returned is at least $\chi(G) \geq 4 \mathrm{~m}$. This gives a polynomial-time algorithm to decide whether $G_{0}$ is 3 -colorable, in contradiction to Lemma 13 unless $\mathrm{P}=\mathrm{NP}$.

\section{Approximation algorithms}

Let us now turn our attention to the additive approximation algorithms. The algorithms we present use ideas similar to the ones of the 2-approximation algorithm of Demaine et al. [2] and of the additive approximation algorithms of Kawarabayashi et al. [9] and Demaine et al. [3]. We find a partition of the vertex set of the input graph $G$ into parts $L$ and $C$ such that $G[L]$ has bounded tree-width (and thus its chromatic number can be determined exactly) and $G[C]$ has bounded chromatic number, and color the parts using disjoint sets of colors. The existence of such a decomposition is proved using the minor structure theorem [12], in the variant limiting the way apex vertices attach to the surface part of the decomposition. The proof of this stronger version can be found in [5]. Let us now introduce definitions necessary to state this variant of the structure theorem.

A tree decomposition of a graph $G$ is a pair $(T, \beta)$, where $T$ is a tree and $\beta$ is a function assigning to each vertex of $T$ a subset of $V(G)$, such that for each $u v \in E(G)$ there exists $z \in V(T)$ with $\{u, v\} \subseteq \beta(z)$, and such that for each $v \in V(G)$, the set $\{z \in V(T): v \in \beta(z)\}$ induces a non-empty connected subtree of $T$. The width of the tree decomposition is $\max \{|\beta(z)|: z \in V(T)\}-1$, and the tree-width of a graph is the minimum of the widths of its tree decompositions.

The decomposition is rooted if $T$ is rooted. For a rooted tree decomposition $(T, \beta)$ and a vertex $z$ of $T$ distinct from the root, if $w$ is the parent of $z$ in $T$, we write $\beta \uparrow z:=\beta(z) \cap \beta(w)$ and $\beta \downarrow z:=\beta(z) \backslash \beta(w)$. If $z$ is the root of $T$, then $\beta \uparrow z:=\emptyset$ and $\beta \downarrow z:=\beta(z)$. The torso expansion of a graph $G$ with respect to its rooted tree decomposition $(T, \beta)$ is the graph obtained from $G$ by adding a clique on $\beta \uparrow z$ for all $z \in V(T)$.

A path decomposition is a tree decomposition $(T, \beta)$ where $T$ is a path. A vortex with boundary sequence $v_{1}, \ldots, v_{s}$ and depth $d$ is a graph with a path decomposition $\left(p_{1} p_{2} \ldots p_{s}, \beta\right)$ such that $\left|\beta\left(p_{i}\right)\right| \leq d+1$ and $v_{i} \in \beta\left(p_{i}\right)$ for $i=1, \ldots, s$. An embedding of a graph in a surface is 2 -cell if each face of the embedding is homeomorphic to an open disk. 
- Theorem 15 (Dvořák and Thomas [5]). For every non-negative integer $t$ and a $t$-apex graph $H$, there exists a constant $a_{H}$ such that the following holds. For every $H$-minor-free graph $G$, there exists a rooted tree decomposition $(T, \beta)$ of $G$ with the following properties. Let $G^{\prime}$ denote the torso expansion of $G$ with respect to $(T, \beta)$. For every $v \in V(T)$, there exists a set $A_{v} \subseteq \beta(v)$ of size at most $a_{H}$ with $\beta \uparrow v \subseteq A_{v}$, a set $A_{v}^{\prime} \subseteq A_{v}$ of size at most $t-1$, and subgraphs $G_{v}, G_{v, 1}, \ldots, G_{v, m}$ of $G^{\prime}\left[\beta(v) \backslash A_{v}\right]$ for some $m \leq a_{H}$ such that

(a) $G^{\prime}\left[\beta(v) \backslash A_{v}\right]=G_{v} \cup G_{v, 1} \cup \ldots \cup G_{v, m}$, and for $1 \leq i<j \leq m$, the graphs $G_{v, i}$ and $G_{v, j}$ are vertex-disjoint and $G^{\prime}$ contains no edges between $V\left(G_{v, i}\right)$ and $V\left(G_{v, j}\right)$,

(b) the graph $G_{v}$ is 2-cell embedded in a surface $\Sigma_{v}$ in which $H$ cannot be drawn,

(c) for $1 \leq i \leq m, G_{v, i}$ is a vortex of depth $a_{H}$ intersecting $G_{v}$ only in its boundary sequence, and this sequence appears in order in the boundary of a face $f_{v, i}$ of $G_{v}$, and $f_{v, i} \neq f_{v, j}$ for $1 \leq i<j \leq m$,

(d) vertices of $G_{v}$ have no neighbors in $A_{v} \backslash A_{v}^{\prime}$, and

(e) if $w$ is a child of $v$ in $T$ and $\beta(w) \cap V\left(G_{v}\right) \neq \emptyset$, then $\beta \uparrow w \subseteq V\left(G_{v}\right) \cup A_{v}^{\prime}$.

Furthermore, the tree decomposition and the sets and subgraphs as described can be found in polynomial time.

Informally, the graph $G$ is a clique-sum of the graphs $G^{\prime}[\beta(v)]$ for $v \in V(T)$, and $\beta(v)$ contains a bounded-size set $A_{v}$ of apex vertices such that $G^{\prime}[\beta(v)]-A_{v}$ can be embedded in $\Sigma_{v}$ up to a bounded number of vortices of bounded depth. Furthermore, at most $t-1$ of the apex vertices (forming the set $A_{v}^{\prime}$ ) can have neighbors in the part $G_{v}$ of $G^{\prime}[\beta(v)]-A_{v}$ drawn in $\Sigma_{v}$, or in the other bags of the decomposition that intersect $G_{v}$. Note that it is also possible that $\Sigma_{v}$ is the null surface, and consequently $A_{v}=\beta(v)$.

We need the following observation on graphs embedded up to vortices.

- Lemma 16. Let $\Sigma$ be a surface of Euler genus $g$ and let a be a non-negative integer. Let $G$ be a graph and let $G_{0}, G_{1}, \ldots, G_{m}$ be its subgraphs such that $G=G_{0} \cup G_{1} \cup \ldots \cup G_{m}$, the subgraphs $G_{1}, \ldots, G_{m}$ are pairwise vertex-disjoint and $G$ contains no edges between them, $G_{0}$ is 2-cell embedded in $\Sigma$, and there exist pairwise distinct faces $f_{1}, \ldots, f_{m}$ of this embedding such that for $1 \leq i \leq m, G_{i}$ intersects $G_{0}$ only in a set of vertices contained in the boundary of $f_{i}$. If the graphs $G_{1}, \ldots, G_{m}$ have tree-width at most $a$, then there exists a subset $L_{0}$ of vertices of $G_{0}$ such that $G_{0}-L_{0}$ is planar and the graph $G_{0}\left[L_{0}\right] \cup G_{1} \cup \ldots \cup G_{m}$ has tree-width at most $26 g+9 m+a$.

Proof. If $\Sigma$ is the sphere, then we can set $L_{0}=\emptyset$; hence, we can assume that $g \geq 1$. Let $G_{0}^{\prime}$ be the graph obtained from $G_{0}$ by, for $1 \leq i \leq m$, adding a new vertex $r_{i}$ drawn inside $f_{i}$ and joined by edges to all vertices of $V\left(G_{0}\right) \cap V\left(G_{i}\right)$. Note that $G_{0}^{\prime}$ has a 2-cell embedding in $\Sigma$ extending the embedding of $G$. Let $F$ be a subgraph of $G_{0}^{\prime}$ such that the embedding of $F$ in $\Sigma$ inherited from the embedding of $G_{0}^{\prime}$ is 2-cell and $|V(F)|+|E(F)|$ is minimum. Then $F$ has only one face, since otherwise it is possible to remove an edge separating distinct faces of $F$, and $F$ has minimum degree at least two, since otherwise we can remove a vertex of degree at most 1 from $F$. By generalized Euler's formula, we have $|E(F)|=|V(F)|+g-1$, and thus $F$ contains at most $2(g-1)$ vertices of degree greater than two. By considering the graph obtained from $F$ by suppressing vertices of degree two, we see that $F$ is either a cycle (if $g=1$ ) or a subdivision of a graph with at most $3(g-1)$ edges.

Let $M_{0}$ be the set of vertices of $F$ of degree at least three and their neighbors. We claim that each vertex of $V(F) \backslash M_{0}$ is adjacent in $G_{0}^{\prime}$ to only two vertices of $V(F)$. Indeed, suppose that a vertex $x \in V(F) \backslash M_{0}$ has at least three neighbors in $G_{0}^{\prime}$ belonging to $V(F)$. Let $w$ and $y$ be the neighbors of $x$ in $F$, and let $z$ be a vertex distinct from $w$ and $y$ adjacent to $x$ in $G_{0}^{\prime}$. The graph $F+x z$ has two faces, and by symmetry, we can assume that the edge $x y$ separates them. Since $x \notin M_{0}$, the vertex $y$ has degree two in $F$, and thus the embedding of $F+x z-y$ is 2-cell, contradicting the minimality of $F$. 
Let $M_{1}$ be the set of vertices of $F$ at distance at most 4 from a vertex of degree greater than two. Note that $\left|M_{1}\right| \leq 26 g$. For $1 \leq i \leq m$, let $N_{i}$ denote the set of vertices of $F-M_{1}$ that are in $G_{0}^{\prime}$ adjacent to a vertex of $V\left(G_{i}\right) \cap V\left(G_{0}\right) \backslash V(F)$. We claim that $\left|N_{i}\right| \leq 9$. Indeed, suppose for a contradiction that $\left|N_{i}\right| \geq 10$ and consider a path $w_{4} w_{3} w_{2} w_{1} x y_{1} y_{2} y_{3} y_{4}$ in $F$ such that $x$ belongs to $N_{i}$ (vertices $x, w_{1}, \ldots, w_{4}, y_{1}, \ldots, y_{4}$ have degree two in $F$, since $\left.x \notin M_{1}\right)$. If $r_{i} \in V(F)$, then let $Q$ be a path in $G_{0}^{\prime}$ of length two between $x$ and $r_{i}$ through a vertex of $V\left(G_{i}\right) \cap V\left(G_{0}\right) \backslash V(F)$; note that $r_{i} \notin\left\{w_{1}, w_{2}, y_{1}, y_{2}\right\}$, since $r_{i}$ has at least 10 neighbors in $V(F)$ and belongs to $M_{0}$ by the previous paragraph and $x \notin M_{1}$. If $r_{i} \notin V(F)$, then there exists a vertex $z \in N_{i} \backslash\left\{x, w_{1}, \ldots, w_{4}, y_{1}, \ldots, y_{4}\right\}$; we let $Q$ be a path of length at most four between $x$ and $z$ passing only through their neighbors in $V\left(G_{i}\right) \cap V\left(G_{0}\right) \backslash V(F)$ and possibly through $r_{i}$. By symmetry, we can assume that the edge $x y_{1}$ separates the two faces of $F+Q$, and the graph $F+Q-\left\{y_{1}, y_{2}\right\}$ if $r_{i} \in V(F)$ or $F+Q-\left\{y_{1}, y_{2}, y_{3}, y_{4}\right\}$ if $r_{i} \notin V(F)$ contradicts the minimality of $F$.

Let $M=\left(M_{1} \cup \bigcup_{i=1}^{m} N_{i}\right) \cap V\left(G_{0}\right)$; we have $|M| \leq 26 g+9 m$. Let $L_{0}=V(F) \cap V\left(G_{0}\right)$. Clearly, $G_{0}-L_{0} \subseteq G_{0}^{\prime}-V(F)$ is planar. Let $G^{\prime}$ be the graph obtained from $G_{1} \cup \ldots \cup G_{m}$ by adding vertices of $M$ as universal ones, adjacent to all other vertices of $G^{\prime}$. The tree-width of $G^{\prime}$ is at most $a+|M| \leq 26 g+9 m+a$. Note that each vertex of $L_{0} \backslash V\left(G^{\prime}\right)$ has degree two in $G_{0}\left[L_{0}\right] \cup G_{1} \cup \ldots \cup G_{m}$, and thus $G_{0}\left[L_{0}\right] \cup G_{1} \cup \ldots \cup G_{m}$ is a subgraph of a subdivision of $G^{\prime}$. We conclude that the tree-width of $G_{0}\left[L_{0}\right] \cup G_{1} \cup \ldots \cup G_{m}$ is also at most $26 g+9 m+a$.

Note that the set $L_{0}$ can be found in polynomial time. For the clarity of presentation of the proof we selected $F$ with $|V(F)|+|E(F)|$ minimum; however, it is sufficient to start with an arbitrary inclusionwise-minimal subgraph with exactly one 2-cell face (obtained by repeatedly removing edges that separate distinct faces and vertices of degree at most 1) and repeatedly perform the reductions described in the proof until each vertex of $V(F) \backslash M_{0}$ is adjacent in $G_{0}^{\prime}$ to only two vertices of $V(F)$ and until $\left|N_{i}\right| \leq 9$ for $1 \leq i \leq m$.

For positive integers $t$ and $a$, we say that a rooted tree decomposition $(T, \beta)$ of a graph $G$ is $(t, a)$-restricted if for each vertex $v$ of $T$, the subgraph of the torso expansion of $G$ induced by $\beta \downarrow v$ is planar, $|\beta \uparrow v| \leq t-1$, and each vertex of $\beta \downarrow v$ has at most $a-1$ neighbors in $G$ that belong to $\beta \uparrow v$. Using the decomposition from Theorem 15, we now partition the considered graph into a part of bounded tree-width and a $(t, t)$-restricted part.

- Theorem 17. For every positive integer $t$ and a $t$-apex graph $H$, there exists a constant $c_{H}$ with the following property. The vertex set of any $H$-minor-free graph $G$ can be partitioned in polynomial time into two parts $L$ and $C$ such that $G[L]$ has tree-width at most $c_{H}$ and $G[C]$ has a $(t, t)$-restricted rooted tree decomposition. Additionally, for any such graph $H$ and positive integers $a \leq b$, there exists a constant $c_{H, a, b}$ such that if $G$ is $H$-minor-free and does not contain $K_{a, b}$ as a subgraph, then $L$ and $C$ can be chosen so that $G[L]$ has tree-width at most $c_{H, a, b}$ and $G[C]$ has a $(t, a)$-restricted rooted tree decomposition.

Proof. Let $a_{H}$ be the constant from Theorem 15 for $H$, and let $g$ be the maximum Euler genus of a surface in which $H$ cannot be embedded. Let $c_{H}=26 g+11 a_{H}$ and $c_{H, a, b}=$ $c_{H}+\left(\begin{array}{c}t-1 \\ a\end{array}\right)(b-1)$.

Since $G$ is $H$-minor-free, we can in polynomial time find its rooted tree decomposition $(T, \beta)$, its torso expansion $G^{\prime}$, and for each $v \in V(T)$, find $A_{v}, A_{v}^{\prime}, G_{v}, G_{v, 1}, \ldots, G_{v, m}$, and $\Sigma_{v}$ as described in Theorem 15. Let $L_{v}^{\prime}$ be the set of vertices obtained by applying Lemma 16 to $G_{v}, G_{v, 1}, \ldots, G_{v, m}$; i.e., $G_{v}-L_{v}^{\prime}$ is planar and the graph $G_{v}\left[L_{v}^{\prime}\right] \cup G_{v, 1} \cup \ldots \cup G_{v, m}$ has tree-width at most $26 g+10 a_{H}$. When considering the case that $G$ does not contain $K_{a, b}$ as a subgraph, let $S_{v}$ be the set of vertices of $G_{v}$ that have at least $a$ neighbors in $G$ belonging to $A_{v}$ (and thus to $A_{v}^{\prime}$ ); otherwise, let $S_{v}=\emptyset$. Since there are at most $\left(\begin{array}{c}t-1 \\ a\end{array}\right)$ ways how to choose a set of $a$ neighbors in $A_{v}^{\prime}$ and no $b$ vertices can have the same set of $a$ neighbors in $A_{v}^{\prime}$, we have $\left|S_{v}\right| \leq\left(\begin{array}{c}t-1 \\ a\end{array}\right)(b-1)$. Let $L_{v}=\left(A_{v} \backslash \beta \uparrow v\right) \cup V\left(G_{v, 1} \cup \ldots \cup G_{v, m}\right) \cup L_{v}^{\prime} \cup S_{v}$. 
We define $L=\bigcup_{v \in V(T)} L_{v}$. Note that $L \cap \beta(v) \subseteq L_{v} \cup(\beta \uparrow v) \subseteq L_{v} \cup A_{v}$. Consequently, $G^{\prime}[L \cap \beta(v)]$ is obtained from $G_{v}\left[L_{v}^{\prime}\right] \cup G_{v, 1} \cup \ldots \cup G_{v, m}$ by adding $S_{v}$ and some of the vertices of $A_{v}$, and consequently $G^{\prime}[L \cap \beta(v)]$ has tree-width at most $c_{H, a, b}$ when considering the case that $G$ does not contain $K_{a, b}$ as a subgraph and at most $c_{H}$ otherwise. The graph $G[L]$ is a clique-sum of the graphs $G^{\prime}[L \cap \beta(v)]$ for $v \in V(T)$, and thus the tree-width of $G[L]$ is also at most $c_{H, a, b}$ or $c_{H}$.

Let $C=V(G) \backslash L$, and consider the graph $G[C]$. For $v \in V(T)$, let $\beta^{\prime}(v)=\beta(v) \cap C$. Then $\left(T, \beta^{\prime}\right)$ is a rooted tree decomposition of $G[C]$ such that for every $v \in V(T)$, the graph $G^{\prime}\left[\beta^{\prime} \downarrow v\right] \subseteq G_{v}-L_{v}^{\prime}$ is planar, all vertices of $\beta^{\prime} \uparrow v$ adjacent in $G$ to a vertex of $\beta^{\prime} \downarrow v$ belong to $A_{v}^{\prime}$ (and thus there are at most $t-1$ such vertices), and when considering the case that $G$ does not contain $K_{a, b}$ as a subgraph, each vertex of $\beta^{\prime} \downarrow v$ has at most $a-1$ neighbors in $G$ belonging to $\beta^{\prime} \uparrow v$.

Note that $\beta^{\prime} \uparrow v$ can contain vertices not belonging to $A_{v}^{\prime}$, and thus $\beta^{\prime} \uparrow v$ can have size larger than $t-1$, and the tree decomposition $\left(T, \beta^{\prime}\right)$ is not necessarily $(t, t)$-restricted. However, by the condition (e) from the statement of Theorem 15, the vertices of $\left(\beta^{\prime} \uparrow v\right) \backslash A_{v}^{\prime}$ can only be contained in the bags of descendants of $v$ which are disjoint from $V\left(G_{v}\right)$, and thus we can fix up this issue as follows.

If $w$ is a child of $v$ and $\beta^{\prime}(w) \cap V\left(G_{v}\right)=\emptyset$, we say that the edge $v w$ is skippable; note that in that case $\beta^{\prime} \uparrow w \subseteq \beta^{\prime} \uparrow v$. For each vertex $w$ of $T$, let $f(w)$ be the nearest ancestor of $w$ such that the first edge on the path from $f(w)$ to $w$ in $T$ is not skippable. Let $T^{\prime}$ be the rooted tree with vertex set $V(T)$ where the parent of each vertex $w$ is $f(w)$. Observe that $\left(T^{\prime}, \beta^{\prime}\right)$ is a tree decomposition of $G[C]$. Furthermore, denoting by $z$ the child of $f(w)$ on the path from $f(w)$ to $w$ in $T$, note that if a vertex $x \in \beta^{\prime} \uparrow f(w)$ is contained in $\beta^{\prime}(w)$, then $x \in \beta(z)$, and since the edge $f(w) z$ is not skippable, the condition (e) from the statement of Theorem 15 implies that $x \in A_{f(w)}^{\prime}$.

Hence, letting $\beta^{\prime \prime}(v)=\left(\beta^{\prime} \downarrow v\right) \cup\left(A_{v}^{\prime} \cap C\right)$ for each vertex $v$ of $T^{\prime}$, we conclude that $\left(T^{\prime}, \beta^{\prime \prime}\right)$ is a rooted tree decomposition of $G[C]$ which is $(t, t)$-restricted, and when considering the case that $G$ does not contain $K_{a, b}$ as a subgraph, the decomposition is $(t, a)$-restricted.

Let us now consider the chromatic number of graphs with a $(t, a)$-restricted tree decomposition.

- Lemma 18. Let $a$ and $t$ be positive integers. Let $G$ be a graph with a $(t, a)$-restricted rooted tree decomposition $(T, \beta)$. The chromatic number of $G$ is at most $\min (t+3, a+4)$. Additionally, if $G$ is triangle-free, then the chromatic number of $G$ is at most $\lceil(13 t+172) / 14\rceil$.

Proof. We can color $G$ using $t+3$ colors, starting from the root of the tree decomposition, as follows. Suppose that we are considering a vertex $v \in V(T)$ such that $\beta \uparrow v$ is already colored. Since $|\beta \uparrow v| \leq t-1$, this leaves at least 4 other colors to be used on $G[\beta \downarrow v]$. Hence, we can extend the coloring to $G[\beta \downarrow v]$ by the Four Color Theorem.

We can also color $G$ using $a+4$ colors, starting from the root of the tree decomposition, as follows. For each vertex $x$ of $\beta \downarrow v$, at most $a-1$ colors are used on its neighbors in $\beta \uparrow v$, leaving $x$ with at least 5 available colors not appearing on its neighbors. Since $G[\beta \downarrow v]$ is planar, we can color it from these lists of size at least 5 using the result of Thomassen [15], again extending the coloring to $G[\beta \downarrow v]$.

Finally, suppose that $G$ is triangle-free. Let $G^{\prime}$ be the torso expansion of $G$ with respect to $(T, \beta)$, and let $c=\lceil(13 t+172) / 14\rceil$. We again color $G$ starting from the root of the tree decomposition using at most $c$ colors. Additionally, we choose the coloring so that the following invariant is satisfied: $(\star)$ for each vertex $w$ of $T$ and for each independent set $I$ in $G[\beta(w)]$ such that $I \cap \beta \downarrow w$ induces a clique in $G^{\prime}$, at most $c-6$ distinct colors are used on $I$. 
Let $v$ be a vertex of $T$. Suppose we have already colored $\beta \uparrow v$, and we want to extend the coloring to $\beta \downarrow v$. Note that the choice of this coloring may only affect the validity of the invariant $(\star)$ at $v$ and at descendants of $v$ in $T$. Consider any descendant $w$ of $T$. Coloring $\beta \downarrow v$ can only assign color to vertices of $\beta \uparrow w$, and since $G^{\prime}$ is the torso expansion of $G$, the set $\beta \uparrow w \cap \beta \downarrow v$ induces a clique in $G^{\prime}$. Consequently, the validity of $(\star)$ at $v$ implies the validity at $w$ (until more vertices of $G$ are assigned colors), and thus when choosing the coloring of $\beta \downarrow v$, we only need to ensure that $(\star)$ holds at $v$.

The graph $G^{\prime}[\beta \downarrow v]$ is planar, and thus it is 5 -degenerate; i.e., there exists an ordering of its vertices such that each vertex is preceded by at most 5 of its neighbors. Let us color the vertices of $\beta \downarrow v$ according to this ordering, always preserving the validity of $(\star)$ at $v$. Suppose that we are choosing a color for a vertex $x \in V(\beta \downarrow v)$. Let $P_{x}$ consist of the neighbors of $x$ in $G^{\prime}$ belonging to $\beta \downarrow v$ that precede it in the ordering; we have $\left|P_{x}\right| \leq 5$. Note that all cliques in $G^{\prime}[\beta \downarrow v]$ containing $x$ and with all other vertices already colored are subsets of $P_{x} \cup\{x\}$. Let $Q_{x}=P_{x} \cup \beta \uparrow v$; we have $\left|Q_{x}\right| \leq t+4$.

Let $N_{x}$ consist of vertices of $Q_{x}$ that are adjacent to $x$ in $G$. We say that a color $a$ is forbidden at $x$ if there exists an independent set $A_{a} \subseteq Q_{x} \backslash N_{x}$ of $G$ such that $A_{a} \cap P_{x}$ is a clique in $G^{\prime}$ and $c-6$ colors distinct from $a$ appear on $A_{a}$. Observe that assigning $x$ a color which neither appears on $N_{x}$ nor is forbidden results in a proper coloring that preserves the invariant $(\star)$ at $v$.

Suppose first that no color is forbidden at $x$. Since $G$ is triangle-free, $N_{x} \backslash P_{x}$ is an independent set in $G[\beta \uparrow v]$, and by $(\star)$, at most $c-6$ colors appear on $N_{x} \backslash P_{x}$. Since $\left|P_{x}\right| \leq 5$, it follows that some color does not appear on $N_{x}$, as required.

Hence, we can assume that some color is forbidden at $x$, and thus there exists an independent set $Z_{1} \subseteq Q_{x} \backslash N_{x}$ of size at least $c-6$ such that vertices of $Z_{1}$ are assigned pairwise distinct colors. Since $\left|Q_{x}\right| \leq t+4$, at most $t+4-(c-6)=t+10-c$ of these colors appear at least twice on $Q_{x}$, and thus there exists a set $Z_{2} \subseteq Z_{1}$ of size at least $c-6-(t+10-c)=2 c-t-16$ such that the color of each vertex of $Z_{2}$ appears exactly once on $Q_{x}$ (and thus does not appear on $N_{x}$ ). Let $Z=Z_{2} \backslash P_{x}$; we have $|Z| \geq 2 c-t-21$. We claim that not all colors appearing on $Z$ are forbidden; since such colors do not appear on $N_{x}$, we can use them to color $x$.

For contradiction, assume that colors of all vertices of $Z$ are forbidden at $x$. Let $Z=\left\{z_{1}, \ldots, z_{m}\right\}$ for some $m \geq 2 c-t-21$, and for $a=1, \ldots, m$, let $a$ be the color of $z_{a}$. Since $a$ is forbidden at $x$, there exists an independent set $A_{a} \subseteq Q_{x} \backslash N_{x}$ such that $A_{a} \cap P_{x}$ is a clique in $G^{\prime}$ and $c-6$ colors distinct from $a$ appear on $A_{a}$. Note that $A_{a} \cup\left\{z_{a}\right\}$ is not an independent set, as otherwise this set contradicts the invariant $(\star)$ at $v$. Hence, we can choose a neighbor $f(a)$ of $z_{a}$ in $A_{a}$. Since $Z$ is an independent set, we have $f(a) \notin Z$. Furthermore, we claim that the preimage in $f$ of each vertex has size at most 6 : if say $f\left(z_{1}\right)=\ldots=f\left(z_{7}\right)=y$, then for $i=1, \ldots, 7$, the vertex $z_{i}$ would have a neighbor $y$ in the independent set $A_{1}$, and thus $z_{1}, \ldots, z_{7} \notin A_{1}$; however, the only appearance of colors $1, \ldots, 7$ in $Q_{x}$ is on the vertices $z_{1}, \ldots, z_{7}$, and thus at most $c-7$ colors would appear on $A_{1}$. We conclude that $|f(Z)| \geq|Z| / 6$, and thus $t+4 \geq\left|Q_{x}\right| \geq|Z|+|f(Z)| \geq \frac{7}{6}|Z| \geq \frac{7}{6}(2 c-t-21) \geq(6 t+25) / 6$. This is a contradiction.

Combining Lemma 18 with Theorem 17 (coloring $G[L]$ using at most $\chi(G)$ colors in linear time [1], using the fact that $G[L]$ has bounded tree-width, and coloring $G[C]$ using a disjoint set of colors), we obtain Theorems 4,7 , and 8. 


\section{References}

1 Stefan Arnborg and Andrzej Proskurowski. Linear time algorithms for NP-hard problems restricted to partial $k$-trees. Discrete Applied Mathematics, 23:11-24, 1989.

2 Erik D. Demaine, MohammadTaghi Hajiaghayi, and Ken-ichi Kawarabayashi. Algorithmic graph minor theory: Decomposition, approximation, and coloring. In 46th Annual IEEE Symposium on Foundations of Computer Science (FOCS'05), pages 637-646. IEEE, 2005.

3 Erik D. Demaine, MohammadTaghi Hajiaghayi, and Ken-ichi Kawarabayashi. Approximation algorithms via structural results for apex-minor-free graphs. Automata, Languages and Programming, pages 316-327, 2009.

4 Matt DeVos, Guoli Ding, Bogdan Oporowski, Daniel Sanders, Bruce Reed, Paul Seymour, and Dirk Vertigan. Excluding any graph as a minor allows a low tree-width 2-coloring. $J$. Comb. Theory, Ser. B, 91:25-41, 2004.

5 Zdeněk Dvořák and Robin Thomas. List-coloring apex-minor-free graphs. arXiv, 1401.1399, 2014.

6 Zdeněk Dvořák, Daniel Král', and Robin Thomas. Three-coloring triangle-free graphs on surfaces VII. A linear-time algorithm. ArXiv, 1601.01197, 2016.

7 Michael Garey and David Johnson. Computers and Intractability: A Guide to the Theory of NP-completeness. WH Freeman \& Co. New York, NY, USA, 1979.

8 Herbert Grötzsch. Ein Dreifarbensatz für dreikreisfreie Netze auf der Kugel. Math.-Natur. Reihe, 8:109-120, 1959.

9 Ken-ichi Kawarabayashi, Erik D. Demaine, and MohammadTaghi Hajiaghayi. Additive approximation algorithms for list-coloring minor-closed class of graphs. In Proceedings of the twentieth Annual ACM-SIAM Symposium on Discrete Algorithms, pages 1166-1175. SIAM, 2009.

10 Alexandr Kostochka. Lower bound of the Hadwiger number of graphs by their average degree. Combinatorica, 4:307-316, 1984.

11 Neil Robertson and Paul D. Seymour. Graph Minors. V. Excluding a planar graph. J. Combin. Theory, Ser. B, 41:92-114, 1986.

12 Neil Robertson and Paul D. Seymour. Graph Minors. XVI. Excluding a non-planar graph. J. Combin. Theory, Ser. B, 89(1):43-76, 2003.

13 Robin Thomas. Conference Graph Theory 2008 at Sandbjerg Manor; slides at http:// people.math.gatech.edu/ thomas/SLIDE/beyondgrot.pdf.

14 Carsten Thomassen. Five-Coloring Maps on Surfaces. J. of Combin. Theory, Ser. B, 59:89-105, 1993.

15 Carsten Thomassen. Every planar graph is 5-choosable. J. Combin. Theory, Ser. B, 62:180$181,1994$.

16 David Zuckerman. Linear degree extractors and the inapproximability of Max Clique and Chromatic Number. Theory of Computing, 3:103-128, 2007. 